\title{
CLINICAL CONSIDERATIONS OF DENTAL IMPLANT SYSTEM IN IMMEDIATE LOADING IMPLANT CASES
}

\author{
Carolina Damayanti Marpaung, Ruth Nadia Susilo, Leonard Nelwan
}

(Private Practitioners)

\begin{abstract}
Immediate loading of dental implant has been researched intensively in the development of Branemark's early concept of 2 stages implant placement. This was embarked from both patients and practitioners convenience towards a simpler protocol and shorter time frame. Many recent researches later found that micromotions derived from occlusal loading for a certain degree, instead of resuiting a fibrous tissue encapsulation, can enhance the osseointegration process. Denta! Implant system enhancement towards maximizing the primary stability held a key factor in Branemark's concept development. Surgical protocol and implant design was found to give a significant contribution to the prognosis of immediate-loading implants. Indonesian Journal of Dentistry 2006; Edisi Khusus KPPIKG XIV:338-342.
\end{abstract}

\section{Introduction}

Dental implant has been widely considered as a predictable rehabilitation treatment since osseointegration concept was introduced by Dr. IV Branemark et al in 1977. His 2-stage implant placement protocol comprises 4-6 months nonloading time frame which became a prerequisite in Dental Implant treatments. ${ }^{1}$ The rationale for such a delay is that premature loading may lead to fibrous tissue encapsulation instead of direct bone apposition. Furthermore, necrotic bone which is derived from surgical trauma at the implant bed border is not capable of load-bearing and must be first replaced by a new bone. ${ }^{2}$ Rapid remodeling of dead bone layer compromises the strength of osseous tissue supporting the bone-implant site which leads to implant failure. However, $80 \%$ of their patients have advanced bone resorption, $10 \%$ with extreme resorption, and the remaining $10 \%$ moderate resorption. All patients have poor bone quality offering unfavorable conditions for the mechanical retention of fixation which provides primary stability. ${ }^{3.4}$ These limitations accounted for the need to delay loading for such a long period.
Later studies were then carried out to shorten the non loading time period through production of new implant designs, development of new technique, and implementation of recently gained knowledge. Based on the theory that mechanical stimulation under bone physiological threshold can have a profound effect on differentiation and development of mesenchymal tissue, immediate loading was then vastly studied. The histological study by Piatelli showed that $60-70 \%$ bone implant contact with immediate loading had no fibrous encapsulation which confirmed the above theory. ${ }^{5.6}$ The ultimate goal of an immediate loading protocol is to reduce the number of surgical interventions and shorten the time frame between surgery and prosthetic delivery, without sacrificing implant success.

Many studies have shown high success rate in immediate loading implants, both clinically ${ }^{7 / 10}$ and experimentally. ${ }^{2.5 .11}$ Ledermann pioneered the clinical studies on 138 patients with 476 dental implants, and showed $91.2 \%$ success rate. ${ }^{3}$ Piatelli's histological study on monkeys confirmed a good bone-implant contact in immediate implant cases which has lead to an assumption that controlled 
romon stimulates bone formation at the ident site. $^{12}$ This was supported by Henry et a 1 round mature and dense cortical bone around - loading implants. ${ }^{13}$ Nonetheless all the mas agree that the protocol must be carried out crusty with strict patient selection. Furthermore, of the studies conducted also came to the enciusion that primary stability is the most marant factor for a good treatment prognosis.

Prumary stability alone has 3 factors: bone, act placement technique, and implant design. E so many dental implant varieties available on market today, practitioners must have a sound redge on the principle of implant morphology, rixirnal. and surface treatment suitable for mrentiate loading cases. The aim of this paper was I review dental implant systems used in clinical Emediate loading studies and get a clear picture of ze principles in operating a dental implant system tor such cases.

\section{Literature Studies}

Ledermann utilized Titanium Plasma Sprayed .PS) endosteal screw implants (Straumann istrute, Waldenburg, Switzerland) in anterior -andible to stabilize overdentures. The protocol alled for 3-4 rough self-tapped implants, long sough to get bicortical anchorage, which was rmediately splinted and loaded on the same day. Fis up to 81 months follow up report showed $91.2 \%$ ruccess rate based on 476 implants placed in 138 zatients, $81 \%$ of the failures occurred during the first $:$ ar. Failures were related to the lack of primary stabiliry, lack of at least $11 \mathrm{~mm}$ of available bone, zuccal and lingual perforation, too early insertion in ir, extraction socket, and insufficient hygiene. ${ }^{3}$

Ledermann's study brought a significant shange in implant treatment perspective. Babbush et al. replicated the Ledermann protocol on implant retained overdentures in 129 patients rehabilitated with 514 implants. Four TPS implants were placed in the interforaminal area of mandible and splinted within 2-3 days by the mean of a dolder bar and ioaded. The 5 years follow up showed $96.1 \%$ success rate. All failures occurred during the first year, whereas $80 \%$ happened during the first 6 months. Failures were related to delayed splinting and perforation of the inferior border with secondary infection. $^{14}$

Chiapasco et al. conducted a multicenter study on the similar protocol as Lederman's. The study incorporated 194 patients with 4 different systems: TPS and ITI screw implants (Straumann Institute, Waldenburg, Switzerland), NLS screw implants (Friatec, Mannheim, Germany) and Ha-Ti screw implants (Mathys Dental Implants, Bettlach, Switzerland). Immediately after the implants placement, a U-shaped gold bar was fabricated and implants were immediately loaded with an overdenture. The study was monitored from a minimum of 2 years to a maximum of 13 years, with a mean follow up of 6.4 years. The overall success rate was $96.9 \%$, whereas the failure rate of bars was $1.5 \%$. No statistically significant difference was found between different implant systems, although performed in 4 different centers. ${ }^{15}$

Current researches using different dental implant systems showed similar results. Lorenzoni et al. reported $100 \%$ success rate on their 6 month study on Frialit-2 implants. The implants were placed in interforaminal region located at positions $34,33,32,42,43$, and 44 . In order to obtain an in-patient comparison of immediate loading and non-loading implants, the ones at 33 and 43 were chosen to be immediately loaded by a dolder-bar retained overdenture. A high intimate bone apposition was seen in the immediate loading implants although with a lower coronal bone level and clinical stability. ${ }^{16}$ Siddiqui et al. also found a good result in immediate loading cases with AdVent Screw dental implants (Zimmmer, Germany). The system came with an intraosseous body, selftapping, triple-lead threads and a combination of HA-coated and blasted surface. ${ }^{17}$

Another study on immediate loading implant in anterior mandible region was conducted by Steenberghe et al., using Branemark Novum protocol (Nobel Biocare, Gothenburg, Sweden) in 50 patients. The protocol called for 3 implants inserted in the symphyseal area, connected by a very rigid horse shoe-shaped titanium bar. A final screw retained prosthetic framework was placed on top of it immediately or within 2 days after surgery. They found $92.7 \%$ success rate on 12 months follow up study, which is lower than the expected rate criteria proposed by Albrektsson and Sennerby $(95 \%){ }^{18,19}$

Immediate loading protocol has also been studied for fixed prosthetic rehabilitations, one of which was a 3 years study conducted by Testori et al. Their study was carried out in 62 patients using the total of 325 dual acid-etched cylindrical screw shaped Ossotite implants (31, West Palm Beach, FL, USA). Five to six implants were inserted in the interforaminal area of mandible with 10 to $18 \mathrm{~mm}$ in length and 3.75 to $6 \mathrm{~mm}$ in diameter utilizing a 32 
Nm torque. Hybrid prostheses were then delivered within 48 hours of implant placement. During the follow up, two implants were lost within the first 2 months which was related to poor bone quality, leaving a $99.4 \%$ success rate. ${ }^{20}$

Similar protocol was conducted by Randow et al. using 5 to 6 MkII fixtures (Nobel Biocare, AB, Goteborg, Sweden) with a minimum length of 10 $\mathrm{mm}$ implanted in the mandible to support a cross arch bridge which was loaded within 20 days following implant installation. During the 18 months follow up, no fixtures have been lost and no clinica! complications have been reported or recorded. Periimplant soft tissues revealed impeccable conditions at all abservations. ${ }^{21}$

Whilst Testori and Randow et al. limited their immediate loading treatment strictly in the anterior mandible, Bischof et al. studied such cases in both jaws. The study incorporated 63 ITI screw dental implants with 4.1 to $4.8 \mathrm{~mm}$ in diameter, 8 to $13 \mathrm{~mm}$ in length, and SLA surface treatment inserted in 18 patients. The implants were connected and loaded with a cross arch bridge within 2 days. At the one year control, the survival rate of immediate loading implants was $98.4 \%$. This study showed no difference in implant stability compared to the delayed loading implants over the first 3 months. The failure was observed in short implants inserted in type 3 bone. ${ }^{22}$

Coincided with Bischof s result, Ericsson et al. found $86 \%$ success rate in his study comparing immediate loading and 2-stage loading protocol to support cross arch bridge in the maxilla and the mandible. Both protocols were carried out employing Branemark implants of $3.75 \mathrm{~mm}$ in diameter and 13 to $18 \mathrm{~mm}$ in length. Two fixtures were lost up to 5 months in function and were subsequently removed, both were in the Maxilla. ${ }^{23}$

\section{Discussion}

The basic principle of immediate loading implant protocol is primary stability, which depends on two factors, host (bone), and external factor (surgical procedure, implant design, and surface treatment).

It has been recommended to perform immediate procedure in dense homologous bone, which can be generally found in the anterior part of the mandible. The cortical lamellar bone may heal with little interim woven bone formation, ensuring good bone strength while healing next to an endosteal implant. Its fine porosity $(<10 \%)$ favors better mechanical interlocking compared to soft cancellous bone that has $80-95 \%$ porosity, which subsequently provides a better stability to withhold any force from immediate loading in maximizing the physiological threshold to accommodate the osseointegration process. ${ }^{24-27}$ On the contrary, the lack of initial stability in type 4 bone results in lower success rates, varying from $50 \%$ to $94 \%{ }^{28-32}$ which was also found by Testory, Bischof, and Ericsson in their studies.

Almost all of the studies on immediate loading implants recommended the use of screw threaded implants which not only minimize the micromotion of the implant due to the increasing surface area, but also to improve initial stability. With a proper surgical procedure, the implant threads ensure the initial interlocking which is essential in immediate loading cases.

Another implant design related factor is the implant length. The benefit of increased length is needed in providing vertical retention to support initial stability of the bone-implant interface. This is more crucial in immediate loading cases since in the early phase of function, dental implant must withstand the forces by itself prior to bone-implant integration. Additionally it is also benefits from the increasing of surface area as concluded by Misch that for every $3 \mathrm{~mm}$ increase in length, the surface area of a cylinder-shaped implant increases by an average of $20-30 \%{ }^{33,34}$

Implant diameter, though critical to withstand the occlusion force concentrated at the crestal bone, does not contribute as much in initial stability. The main benefit of using wide diameter implant is the increasing surface area as each $0.25 \mathrm{~mm}$ increase in diameter improves functional area support to more than $5-10 \%{ }^{35}$

Surface treatment has been proven to improve initial stability and bone healing process. ${ }^{36-38}$ It benefits from both the rough implant surface and the osteoconductive media given on the surface. An appropriate micro-roughened surface is proven to engage and hold the fibrin strands which are formed during the initial clotting process following the insertion of implant fixtures. The stabilization of the initial clot is mandatory as the fibrin strands form a bridge for the migration of osteoblastic cells from the surrounding osteotomy onto the implant surface. Favorable surface characteristic have also been shown to aid the absorption of proteins, accumulate and activate platelets in providing platelet derived growth factors, thus promoting angiogenesis, and 
differentiate progenitor cells into mature $\rightarrow$ oblasts.

In principle, there are 2 kinds of surface reament: additive and reductive surface. Implants - ${ }_{-2}$ Titanium Plasma Sprayed (TPS) and HA Examg fall into the first category. Pure Titanium aracle which is sprayed through a plasma flame at $200^{\circ} \mathrm{C}$ is shown to increase the surface area by 6 $\because$ imes by the apposition of a porous layer $20-50$ In thick. On the other hand HA coating has been Xiven to accelerate osseointegration. TPS implants Fe still widely used for immediate loading cases sone they showed a very high success rate. The zin concern for both surface treatment is the ventual detachment with subsequent periimplantitis sowed in some studies with HA coating. $33,38-40$

Reductive surface treatment is the development $\because$ the two surfaces discussed to minimize the risk of siface detachment. The micro-roughening process if the titanium implant surface is done by electrorishing, acid-etching, titanium oxide blasting and iast-etching method.39.40 Studies on implants with Eutuctive surface showed a high success rate, though jesn't differ significantly with the additive.

Surgical protocol plays an important role since jental implant procedure is technique sensitive. Proper surgical protocol in an implant system should aclude adequate cooling to avoid the heat generated turing bone drilling. It is recommended to keep the :emperature below $47^{\circ} \mathrm{C}$ to avoid bone necrosis, which could detriment the osseointegration process following a good primary stability. This can be achieved by increasing the speed and torque together to allow a more efficient cutting with no significant ncrease in temperature. ${ }^{33}$

A proper orientation of osteotome instruments to create an undersized preparation of implant site prior to placement is also essential in immediate joading cases. Shallabi proposed the theory supporting this protocol that a lot of small bone fragments are created and pressed in between the rabecular voids and between the screw threads Juring implant installation, hence ensuring a good primary stability and inducing new bone formation. ${ }^{4 \mathrm{I}}$

\section{Conclusion}

Dental implant systems used in immediate loading cases must have the properties that support maximum primary stability. This is to ensure that micromotion can be controlled under physiological threshold to induce a mechanical stimulation on mesenchym tissue. The properties must include screw threaded cylindrical implants, with maximum length and diameter, adequate surface treatment, atraumatic and undersized implant bed preparation.

\section{Reference}

1. Branemark Pf. Osseointegration and Its Experimental Background. J Prosthet Dent 1983: 50: 399410

2. Sagara M, Akagawa $Y$, Nikaj $H$, Tsuru $H$. The Eftect of Early Loading on One-Stage Titanium Alloy Implants in Beagle Dogs: A Pilot Study. J Prosthet Dent 1993; 69: 2818

3. SzmukIer-Moncler \$, Piatelli A, Favero GA, Dubruille JH. Considerations Preliminary to the Application of Early and Immediate Loading Protocols in Dental Implantology. Clinical Oral Implant Researches 2000; 1(1): 12-25

4. Herrera-Briones FJ, Romero-OJid MN, Vallecillo-Capilla M. Update on Immediate Implant Loading: A Review of the Literature. Med Oral 2004; 9(1): 74-81

5. Piattelli A, Paolantonio M, Corigliano M, Scarano A Immediate Loading of Titanium Plasma Sprayed ScrewShaped Impiants in Man: A Clinical and Histological Report of Two Cases. J Periodontol 1997; 68: $591-7$

6. Barone A Covani $U$, Comelini $R$ Ghetlone $E$. Radiographic Bone Density around Immediately Loaded Oral Implants. Clinical Oral Implant Research 2003; 14: 610-5

7. Schnitman PA, Wohrie, Rubenstein JE. Immediate Fixed Interim Prosthesis Supported by Two Stage Threaded Implants: Methodology and Result. Journal of Implantology 1990; 2: 96-105

8. Salama $H$, Rose LF, Salama $M$, Betts NJ. Immediate Loading of Bilaterally Splinted Titanium Root Form Implants in Fixed Prosthodontic: A Technique ReExamined. International Journal of Periodoniology and Restonative Dentistry 1995; 15 : $345-61$

9. Spiekermann H, Jansen VK, Richter EJ, A 10-year FollowUp Study of IMZ and TPS Implants in the Edentulous Mandible Using Bar-Retained Overdentures. Intemational Journal of Oral and Maxillofacial Implants 1995; 10: 23143

10. Chiapasco M, Gatti C, Rosși E, Haefliger W, Markwalder TH. Implant Retained Mandibular Overdentures with Immediate Loading: A Retrospective Multicenter Study on 226 Consecutive Cases. Clinical Oral Implant Research $1997 ; 8$ (I): 48-57

11. Corso M, Sirota C, Fiorellini J, Rasool F, SzmuklerMoncier S, Weber HP. Evaluation of The Ossecintegration of Early Loaded Free-Standing Dental Implants with Various Coatings in the Dog Model: Periotest and Radiographic Results. J Prosthet Dent 1999; 82: 428-35

12. Piatelli A, Corrigliano M, Scarano A, Quaranta M. Bone Reactions to Early Occlusal Loading of Two-Stage Titanium Plasma Sprayed Implants: A Pilot Study in Monkey. International Journal of Periodontology and Restorative Dentistry 1997; 17: 163-9

13. Henry $P$, Tan AE, Leavy J, Johansson CB, Albrektsson $T$. Tissue Regeneration Bony Defects Adjacent to lmmediate Load Titanium Implants Placed in Extraction Sockets: A Study in Dogs. Iniernational Journal of Oral and Maxillofocial Implants 1997; 12: 758-66 
14. Babbusch C. Titanium Plasma Sprayed Screw Implants for the Reconstruction of the Edentulous Mandible. $J$ Orai Maxillofac Surg 1986; 44: 274-82

15. Chiapasco M. Early and Immediate Restoration and Loading of Implants in Completely Edentulous Patients. International Joumal of Oral and Maxillofacial Implants 2004: 19 (Suppl): 76-9l

16. Lorenzoni $M$, Perti $C$, Zhang $K$, Wegscheider WA. Inpatient Comparison of Immediately Loaded and NonLoaded Implants within 6 Months. Clinical Oral Implant Research 2003; 14:273-9

17. Siddigui AA, lsmail JYH, Kukunas S. immediate Loading of Dental Implants in the Edentulous Mandible: A Preliminary Case Report from An International Prospective Multicenter Study. Compendium of Continuing Education in Dentistry 2001; 22 (10): 1-8

18. Steenberghe D, Molly L, Jacobs $\mathrm{R}$, Vandekerckhove $\mathrm{B}$ Quirynen M, Naer 1. The Immediate Rehabilitation by Means of A Ready-Made Final Fixed Prosthesis in The Edentulous Mandible: A l-Year Follow-Up Study on 50 Consecutive Patients. Cfinical Oral Implant Research 2004; 15: $360-5$

19. Albrektsson T, Sennerby L. State of the Art in Orai Implants. J Clin Periodontol 1991; 18: 474.81

20. Testori T, Meltzer A, Fabbro MD, Zuffeti F, TroianoM, Francetti L, Weinstein RL. Immediate Occlusal Loading of Osseotite inplants in the Lower Edentulous faw. A Multicenter Perspective Study. Clinical Oral Implant Research 2004; 15: 278-84

21. Randow $K$, Ericsson I, Nilner $K$, Petersson A, Giantz $P$. Immediate Functional Loading of Branemark dental Implants: An 18-Month Clinical Follow-Up Study. Clinical Oral Implant Research 1999; 10: 8-15

22. Bischof $M$, Nedir R, Szmukier-Moncler $S$, Bernard J, Samson J. Implant Stability Measurement of Delayed and Immediately Loaded Implants during Healing. Clinical Oral Implant Research 2004; 15 : 529-39

23. Ericsson I, Nilson $H$, Lindh $T$, Nilner $K$, Randow $K$ Immediate Functional Loading of Branemark Single Tooth Implants: An 18 Month' Clinical Pilot Follow-Up Study. Clinical Oral Implant Research 2000; 11 (1): 26-33

24. Meredith N. Assessment of Implant Stability as A Prognostic Determinant. IJ Prosthod 1998; Il: 491-501.

25. Friberg B, Sennerby L, Linden B, Grondahl K, Lekholm U. Stability Measurement of Ore-Stage Branemark Implants during Healing in Mandible: A Clinical Resonance Frequency Analysis Study. International Journal of Oral and Maxillofacial Implants $1988 ; 28: 266-72$

26. Balleri P, Cozzolino A, Ghelli L, Monicchioli G, Varriale T. Stability Measurements of Osseointegrated Implants Using Osstell in Partialły Edentulous Jaws after ] Year of Loading: A Pilot Study. Clinical Implant Dentistry and Related Research 2002; 4: 128-32

27. Friberg B, Sennerby L, Meredith N, Lekholn U. A Comparison between Curting Torque and Resonance Frequency Measurements of Maxillary Implants: A 20 Months Clinical Study. International Journal of Oral and Maxillofacial Surgery 1999; 28: 297-303

28. Enquist G, Bergendal T, Kallus $T$ \& Linden $U$. A Retrospective Multicenter Evaluation of Osseointegrated
Implant-Supported Overdentures. International Jowrnal of Oral and Maxillofacial Implants 1988; 2: 129-34

29. Jaffin RA and Berman CL. The Excessive Loss of Branemark Fixtures in Type IV Bone. A 5-Year Analysis. $J$ Periodontol 1991; 62: 2-4

30. Johns RB, Jemt T Heath MR. A Multicenter Study of Overdentures Supported by Branemark implants. International Journal of Oral and Maxillofacial Implants 1992; 7 : $513-22$

31. Hutton JE, Heath MR, Chai JY, Harnett J, Jemt T, Johns RB, McKenra S, McNamara DC, Van Steenberghe D, Taylor R, Watson RM, Hermann I. Factors Related to Success and Faiłure Rates at A 3-Year Follow Up in A Multicenter Study of Overdentures Supported by Branemark Implants. International Journal of Oral and Maxillofacial Implants 1995; 10: 33-42

32. Jemt T. Implant Treatment in Resorbed Edentulous Upper Jaws. A 3-Year Follow Up Study on 70 Patients. Chinical Oral Implant Research 1993; 4: 187-94

33. Misch C. Dental Implant Prosthetics. St Louis Missouri: Elsevier Mosby. 2005; 322-48

34. Derbabian K, Simonian K. Immediate Loading of Dental Implants: Overview and Rationale. Can Dent Assoc $/ 2005$; 33 (4): $337-41$

35. Jungner $\mathrm{M}$, Lundqvist $\mathrm{P}$, Lundgren $\mathrm{S}$. Oxidized Titanium Implants (Nobel Biocare Tiunite) Compared With Tumed Titanium Implants (Nobel Bicare Mark III) with Respect to Implant Failure in A Group of Consecutive Patients Treated with Early Functional Loading and Two-Stage Protocol. Clinical Oral Implant Research 2005; 16: 308-12

36. Karlsson U, Gotfredsen K, Olsson C. A 2-Year Report on Maxillary and Mandibular Fixed Partial Dentures Supported by Astra-Tech Dental Implants. A Comparison of 2 Implants. Clinical Oral Implant Research 1998; 9: 235-42

37. Ivanoff $\mathrm{CJ}$, Widmark $\mathrm{G}$, Johansson $\mathrm{C}$, Mennerberg A Histologic Evaiuation of Bone Response to Oxidized and Turned Titanium Microimplants in Human Jawbone. Internationat Journal of Oral and Maxillofacial Implants 2003; $18: 341-8$

38. Buser D, Nydegger T, Oxiand TR, Schenk RK, Cochran Di, Snetivy D. Nolte LP. The Interface Shear Strength of Titanium Implants with A Sandblasted and Acid-Etched Surface. A Biomechanical Study in the Maxilla of Miniature Pigs. Jotunal of Biomedical Material Research 1999; 45: $75-83$

39. Maeztu MA Alava Jl, Gay-Escoda C. Ion Implantation: Surface Trealment for Improving the Bone lntegration of Titanium and Tl6A14V Dental Implants. Clinical Oral Implant Research 2003; 14: 57-62

40. Cochran DL, Nummikoski PV, Higginbottom FL, Hermann JS, Makins SD, Buser D. Evaluation of An Endosseous Titanium Implant with A Sandblasted and Acid-Etched Surface in the Canine Mandible: Radiographic Results. Clinical Oral Implant Research 1996: 7 (3): 240-52

41. Shaliabi MM, Wolke JG, Jansen JA. The Effects of Implant Surface Roughness and Surgical Technique on Implant Fixation in An in Vitro Model. Ctimeal Oral Implant Research 2006: 17 (2): 172-8 